\title{
The Effectiveness of a Pelvic Floor Muscle Exercise Program on Urinary Incontinence Following Childbirth: A Systematic Review
}

\author{
Robin Watts, RN BA MHSc PhD FRCNA ${ }^{1}$ \\ Gaby Haddow, PhD BAppSci(Hons) ${ }^{2}$ \\ Jeanette Robertson, RN MSc FRCNA ${ }^{3}$
}

1. Professor of Nursing, Curtin University of Technology, Director, The Western Australian Centre for Evidence Based Nursing and Midwifery a collaborating centre of the Joanna Briggs Institute.

2. Research Project Officer, Library, Curtin University of Technology

3. Curtin University of Technology, Deputy Director, The Western Australian Centre for Evidence Based Nursing and Midwifery a collaborating centre of the Joanna Briggs Institute.

\section{Executive Summary}

\section{Objectives}

The primary objective of this review was to determine from the available evidence, the effectiveness of an antenatal and/or a post natal program of Pelvic Floor Muscle Exercises (PFME) compared with usual care on preventing, reducing or resolving the incidence and severity of stress incontinence, urge incontinence or mixed stress and urge urinary incontinence following childbirth. Secondary objectives were included to examine the effectiveness of a PFME program on pelvic floor muscle strength and on encouraging adherence to an exercising program.

\section{Inclusion Criteria}

\section{Types of studies}

Randomised controlled trials (RCTs) and non-randomised controlled trials (non-RCTs) were included in the review if, in relation to urinary incontinence, and/or adherence to a PFME program, and/or pelvic floor muscle strength, the following had been explored:

- Antenatal PFME compared with usual care

- Postnatal PFME compared with usual care

- A PFME program compared with usual care

Usual care is commonly used to describe the care women normally receive from health professionals in the antenatal and/or postnatal period. In some cases usual care includes a standard information package given to all women attending the health service and in others it is advice about performing PFME.

\section{Types of participants}

Participants included women who experienced a spontaneous onset of labour and who subsequently delivered at more than 20 weeks gestation either vaginally, both spontaneous and assisted, or by non-elective caesarean section.

Exclusions

Women who delivered by elective caesarean section.

Women experiencing postpartum overflow urinary incontinence.

Types of interventions

a) Pelvic floor muscle exercises 
b) PFME instruction and a PFME program's components, such as educational materials, feedback (including biofeedback, eg. information about strength of pelvic floor muscle contractions by various means) and number of PFME.

Exclusions:

- Electrical stimulation of pelvic floor muscles

- Vaginal cones, or

- Other adjunct therapies

In studies that included a subgroup treated with one of these interventions, the results of the subgroup were excluded from the review's analysis.

\section{Types of outcome measures}

Outcomes that were of interest:

- Non-occurrence of urinary incontinence following childbirth

- A change in the frequency, duration, or severity (as appropriate) of urinary incontinence up to twelve months following childbirth.

- A change in the strength of pelvic floor muscle contractions

- Period of time PFME continued after initial instruction

- Frequency of PFME undertaken

- Women's awareness of the importance of PFME

- Satisfaction with PFME instruction.

\section{Search Strategy}

All major electronic sources of information relevant to the topic (eg. PubMed, CINAHL, and the Cochrane Library) were searched to identify published and unpublished studies and previous work in the field. Printed journals were hand-searched and reference lists checked for potentially useful research. The review included any studies undertaken between 1981 and 2003. The search did not attempt to locate unpublished research prior to 1991.

\section{Assessment of Quality}

An independent Review Panel carried out quality assessment of studies. Two members of the panel, using quality assessment checklists developed for the review, reviewed each study.

Disagreements between reviewers were resolved through discussion or a third reviewer examining a study.

\section{Data extraction and analysis}

A data extraction tool was developed to extract data relating to participant characteristics, study methods, interventions, and outcomes. Two reviewers independently extracted the required data.

Randomised controlled trials included in the review were pooled in several meta-analyses using RevMan software programme. Heterogeneity between studies was determined to ensure that they were sufficiently similar to allow for the pooling of their results. Non-randomised controlled trials were discussed in narrative comparisons.

\section{Results}

Six RCTs met the inclusion criteria for the primary objective of the systematic review. The results of this review indicate that antenatal PFME and postnatal PFME are effective in resolving or reducing urinary incontinence following childbirth. There was insufficient evidence to conclude that PFME can prevent urinary incontinence in postpartum women. In most of these studies women were selected randomly and therefore included women without urinary incontinence and women with urinary incontinence. Two RCTs selected their sample on the criteria of existing postpartum urinary incontinence. A subgroup analysis of these studies showed that postnatal PFME also have a significant effect on reducing or resolving urinary incontinence in women with existing postpartum urinary incontinence. 
Four RCTs and four non-RCTs met the inclusion criteria for the secondary objectives of the review. Findings of the studies included in the review suggest a PFME program will improve the frequency with which women perform PFME. Two studies found that women receiving the intervention (a PFME program) and who were performing PFME regularly in the month before data collection were significantly less likely to have any incontinence. The review's results support previous findings showing there is little evidence that a high-intensity PFME program is more effective than a low intensity PFME regimen of exercising. No conclusions about the effectiveness of feedback to a woman about pelvic floor muscle strength, for example perineometer measures, as part of a PFME program can be reached.

The mixed results of this review mean that no conclusions can be reached about the effectiveness of a PFME program, antenatal or postnatal, on improving pelvic floor muscle strength.

A number of studies reported a high percentage of women lost to follow-up and the data collected in most of the studies relied on self-reports relating to urinary incontinence and/or frequency of exercising. These factors may have affected the overall results of the review. However wherever possible, tests for heterogeneity were carried out to determine if studies should be combined in meta-analyses and in other cases the results' limitations are acknowledged.

\section{Implications for Practice}

In terms of the effectiveness of PFME programs, the results of this review indicate that urinary incontinence following childbirth can be improved by performing PFME and that any form of a specific PFME program appears to improve exercising frequency. However, the value of individual components of PFME programs, such as take home materials, reminder telephone calls, and feedback of exercising effectiveness are less clear.

- Encourage women to undertake both antenatal and postnatal PFME (E1).

- Pay particular attention to women with antenatal and postnatal urinary incontinence in providing advice and PFME instruction (E1).

- To encourage adherence and continuation, PFME education programs should be multifaceted with a number of components, rather than only supplying an information booklet (E4).

- Include PFME as a specific program in all antenatal and postnatal care, incorporating at least two individual instructions sessions into the program (E1).

- Provide postpartum contact, particularly for those discharged early, either by telephone, electronic or home visits (E4).

- Design PFM home exercise programs that are realistic given the demands on a mother and that can be incorporated into her daily routine in terms of number and frequency. Two or more training sessions per week are recommended (E4).

- Health professionals working with women in the postpartum period should ask about symptoms of incontinence to ensure assistance is offered to those experiencing urinary incontinence (E4).

\section{Background}

Urinary incontinence is defined by the International Continence Society (ICS) as 'the involuntary loss of urine that is a social or hygienic problem..$^{(1)}$ Urinary incontinence can and does pose significant problems for a woman, impacting on the quality of her physical, social, emotional and sexual life.

Three types of urinary incontinence (stress, urge and mixed) are most frequently discussed in the literature and studied by researchers. The ICS has published definitions for each type. Stress urinary incontinence is 'involuntary leakage on effort or exertion, or on sneezing or coughing', urge urinary incontinence is 'involuntary leakage accompanied by or immediately preceded by 
urgency', and mixed urinary incontinence is 'involuntary leakage associated with urgency also with exertion, effort, sneezing or coughing'. (1)

Childbirth is one of the major causes of urinary incontinence in women. ${ }^{(2-5)} \mathrm{A}$ number of comprehensive papers about the physiological aspects of urinary incontinence and its prevalence are available for further information. ${ }^{(2,4,6-8)}$ Studies of incontinence in the post partum period report prevalence between 6 and 43\%. ${ }^{(6,9-13)}$ For some women urinary incontinence after childbirth is temporary, but others suffer longer-term urinary incontinence problems. In one study, over $60 \%$ of women who experienced stress urinary incontinence during pregnancy reported stress urinary incontinence fifteen years later. ${ }^{(7)}$ The cause of urinary incontinence following childbirth has been associated with a number of factors, including urinary incontinence during the antenatal period, obesity, long second stage of labour, delivery method, size of baby, and the number of previous pregnancies. Researchers attempting to determine the major risk factors for developing urinary incontinence during pregnancy have reported mixed findings, particularly in relation to parity and the size of a baby. ${ }^{(8,14)}$

The teaching of pelvic floor muscle exercises (PFME) is aimed at helping the woman to regain control over the peri-vaginal musculature. Kegel first described these exercises in 1948 after finding that most women with stress urinary incontinence had pelvic floor muscle insufficiency. ${ }^{(15)}$ Kegel argued that restoring the function of the pelvic floor would in turn increase the urethral closure pressure, thus preventing involuntary loss of urine. ${ }^{(16)}$

A number of studies in recent years have sought to measure the effectiveness of PFME. A systematic review of 11 RCTs assessed physical therapies in the treatment and prevention of stress urinary incontinence (SUI) in women and found that there was strong evidence to suggest PFME were effective in reducing the symptoms of SUI. ${ }^{(17)}$ There was limited evidence for the efficacy of high-intensity versus a low intensity regimen of exercising. ${ }^{(18)}$ The low intensity regimen involved performing a specified number of PFME every day at home. For the high intensity regimen participants performed the same home exercises as well as attending weekly exercise training with an instructor. The systematic review also concluded there was evidence that PFME with biofeedback, for example perineometer measures, were more effective than PFME alone. In prevention of SUI, the efficacy of PFME with or without other adjuncts is uncertain.

A systematic review of 43 studies of pelvic floor muscle training for urinary incontinence (stress, urge and mixed) in women by the Cochrane Collaboration ${ }^{(19)}$ found that this training appeared to be an effective treatment of adult women with stress or mixed incontinence and was better than no treatment or placebo. However, methodological problems limited the confidence that could be placed in the results of the review.

In the reviews discussed above the participants in the studies included adult women of all ages. This review is the first to focus on the effectiveness of a PFME program in relation to postpartum urinary incontinence and included women reporting stress, urge and mixed urinary incontinence.

\section{Objectives}

The primary objective of this review was to determine from the available evidence the effectiveness of antenatal and/or post natal PFME compared with usual care for urinary incontinence (stress, urge, mixed) following childbirth. Secondary objectives were included to examine the effectiveness of a PFME program on pelvic floor muscle strength and encouraging exercising adherence.

The specific questions asked were:

1. Do antenatal PFME prevent or reduce urinary incontinence following childbirth? 
2. Do PFME following childbirth resolve or reduce subsequent urinary incontinence?

3. Are PFME effective in improving pelvic floor muscle strength?

4. What is the most effective type of instruction of PFME to encourage adherence to a PFME program?

\section{Criteria for considering studies for this review}

The methods for conducting the systematic review and for assessing the quality of the evidence are based on the work of the Cochrane Collaboration ${ }^{(20)}$ and the Centre for Reviews and Dissemination at the University of York. ${ }^{(21)}$

\section{Types of studies}

Randomised and non-randomised controlled trials studying any or a combination of the questions posed in the review's objectives were sought for inclusion. Studies were included in the review if, in relation to urinary incontinence, adherence to a PFME program, and/or pelvic floor muscle strength, the following had been explored:

- Antenatal PFME compared with usual care

- Postnatal PFME compared with usual care

- A PFME program compared with usual care

Usual care is commonly used to describe the care women normally receive from health professionals in the antenatal and/or postnatal period. In some cases usual care includes a standard information package given to all women attending the health service and in others it is advice about performing PFME.

Studies that included women with postpartum overflow urinary incontinence were excluded from the review.

In respect to locating studies about the effectiveness of antenatal or postnatal PFME on urinary incontinence (objectives 1 and 2), the review focused on identifying randomised controlled trials. The more qualitative nature of measuring the effectiveness of a PFME program on encouraging adherence and improved pelvic floor muscle strength meant that studies using non-randomised controlled trials and descriptive research methods were also considered for objectives 3 and 4 of the review.

Because the factors, considered by researchers, associated with urinary incontinence have not changed significantly during the last two decades, and because PFME have been proposed as a useful therapy since 1948, the review included any studies undertaken between 1981 and 2003 . The search did not attempt to locate unpublished research prior to 1991 as very few tools exist that provide access to these studies.

Types of participants

Participants included women who experienced a spontaneous onset of labour and who subsequently delivered at more than 20 weeks gestation either vaginally, both spontaneous and assisted, or by non-elective caesarean section. Given the objectives of the review, both women with and without urinary incontinence symptoms were encompassed by the sampling methods of included studies.

Exclusions:

Women who had an elective caesarean section. Studies have demonstrated that there is a lower prevalence of incontinence in women who have undergone an elective caesarean section as no stress has been placed on the pelvic floor muscles. ${ }^{(6)}$ Studies that included women experiencing postpartum overflow urinary incontinence were also excluded from the review. 
Types of interventions

a) Pelvic floor muscle exercises

b) PFME instruction and a PFME program's components e.g. timing, method, content.

Exclusions:

Interventions that included:

- Electrical stimulation of pelvic floor muscles

- Vaginal cones, or

- Other adjunct therapies

For information about the effectiveness of these and surgical interventions for stress urinary incontinence a recent publication in Clinical Evidence is available. ${ }^{(22)}$ In studies that included a subgroup treated with one of these interventions, the results of the subgroup were excluded from the review's analysis.

\section{Types of outcome measures}

Outcomes that were of interest for objectives 1 and 2 included:

- Non-occurrence of urinary incontinence following childbirth

- A change in the frequency, duration, or severity (as appropriate) of urinary incontinence up to twelve months following childbirth.

Outcomes that were of interest for objectives 3 and 4 included:

- Improved strength of pelvic floor muscle contractions

- Period of time PFME continued after initial instruction

- Frequency of PFME undertaken

- Women's awareness of the importance of PFME

- Satisfaction with PFME instruction.

\section{Search strategy for identification of studies}

To identify all relevant studies for the review, the search strategy included searches of:

- Indexes of periodical articles (eg. CINAHL, MEDLINE/PubMed, Current Contents, Science Direct),

- Indexes of reports, theses, and conference papers (eg Dissertation Abstracts, Conference Papers Index, National Research Register - UK),

- Major sources of evidence-based practice information (eg. Cochrane Collaboration, NHS Centre for Research and Dissemination, Agency for Healthcare Research Quality, and clinical trials databases), and

- Web sites of nursing and midwifery organisations and other relevant agencies (eg. MIRIAD - a database of midwifery resources, International Continence Foundation, Society of Urologic Nurses).

Individual strategies were developed for each source searched, adopting the different terminology of index thesauri and/or using the natural language terms of the topic. These strategies were undertaken because it has been established that difficulties can arise when using MeSH terms only to locate clinical trials. ${ }^{(23)}$ The main terms and search strategies used to located studies are listed in Appendix I.

In addition, journals were hand searched, either online or in hard copy form, and search alerts for the topic were established to ensure that relevant papers were identified as they were published. To ensure that all recently published papers had been located, the most relevant journals were individually searched for the publication years of 2001 and 2002 at least. Most of this hand searching was carried out over a longer publication period. For a list of all the major sources 
searched and journals included in the search alerts see Appendix II. The reference lists of relevant papers and research studies were examined for potentially useful links to research not previously located.

The importance of contacting experts in the field to ensure all relevant studies are located has been reported. ${ }^{(24)}$ In this review contact was made with experts who had been involved in systematic reviews about related topics and authors of highly relevant studies to the review.

All relevant studies with an English language abstract were located for assessment against the inclusion criteria.

\section{Methods of the review}

Three primary reviewers and a Review Panel conducted the review. The primary reviewers developed the review protocol and critical appraisal tools, assessed studies against inclusion criteria, coordinated meetings and discussions of the Review Panel, extracted data and wrote the review report. The Review Panel was comprised of 12 nurses and midwives working in the major women's health services in Western Australia, government and higher education. Members of the Panel provided expertise about clinical aspects of the review, piloted the assessment tool, and assessed studies for quality. When reviewing the draft report a consumer representative joined the Panel. One of the primary researchers conducted a workshop for Panel members on critical appraisal prior to the quality assessment of studies being undertaken.

Studies identified in the search were assessed independently for inclusion in the review by two of the primary reviewers. The criteria for inclusion in the review (see Appendix III) were based on types of participants, interventions and outcomes as discussed above.

Studies were not excluded from the review on the basis of research methodology at this stage in the review.

\section{Assessment of methodological quality of studies}

Full text (or abstracts if full text was not available) copies of the studies that met the inclusion criteria were subsequently assessed for methodological quality by two members of the Review Panel. Methodological quality of randomised controlled trials (RCT) which studied the effectiveness of PFME on urinary incontinence following childbirth was assessed using a checklist (see Appendix IV) created for this review, and based on the work of the Cochrane Collaboration ${ }^{(20)}$ and the Centre for Reviews and Dissemination at the University of York. ${ }^{(21)}$ The checklist, piloted with the Review Panel and subsequently modified for layout and wording, required reviewers to assess each study for relevance, scientific merit and bias.

A second methodological quality assessment tool was developed for non-RCT studies that were being considered for inclusion for objectives 3 and 4 of the review. This checklist, at Appendix $V$, was also specifically developed for this review; drawing on information from the Agency for Healthcare Research and Quality report 'Systems to Rate the Strength of Scientific Evidence', and required reviewers to asses each study for scientific merit. ${ }^{(25)}$ Included studies required positive responses to $60 \%$ of the relevant checklist questions.

Methodological quality assessments of studies were discussed at review panel meetings. On three occasions there was uncertainty about the quality of a study or disagreement between assessments and these were resolved through discussion or by a third assessment. Reviewers were not blinded to the studies' author names, affiliations or source. Assessment of quality commenced with the studies (randomised and quasi-randomised controlled trials) related to objectives 1 and 2 of the review. Once these were completed, those studies addressing the other outcomes of interest (objectives 3 and 4 ) were subjected to quality assessment.

For four studies the reviewers required clarification of study methods or additional information 
before a decision could be made about the methodological quality of the study. ${ }^{(26-29)}$ In these cases the authors were contacted. Two authors were unable to provide the additional information required and the studies were subsequently excluded from the review. ${ }^{(28,29)}$ As a result of the quality assessment process, 11 of the 22 studies assessed by the Review Panel were included in the review.

\section{Data extraction}

Data from the RCTs included in the urinary incontinence outcome of the review were extracted independently by two of the primary reviewers using a data extraction tool (see Appendix $\mathrm{VI}$ ) developed and pilot tested for this review. For this part of the review dichotomous data relating to whether women reported experiencing urinary incontinence was extracted from each study. A second data extraction form was developed for the studies included in the other outcomes of interest in the review (see Appendix VII). Both dichotomous and continuous data were extracted from the studies examining the effectiveness of a PFME program on encouraging exercising adherence, and continuous data were extracted from studies looking at pelvic floor muscle strength. Any differences between the reviewers were resolved by discussion. The data extraction tools collected information about each study such as type of design, details of randomisation (if used), study population, intervention; outcomes as listed above, and quality of study assessment. Additional data were required for three studies and the researchers were contacted to obtain the data needed for the study to be included in the review. ${ }^{(27,30,31)}$

\section{Data analysis}

Dichotomous data extracted to examine effectiveness of PFME on postpartum urinary incontinence, were pooled in two meta-analyses using the software program RevMan. One metaanalysis was carried out for the studies that had examined the effectiveness of antenatal PFME and the other for studies about the effectiveness of postnatal PFME. A sub-group analysis was carried out for two studies about postnatal exercises because the study participants were sampled specifically on the basis of pre-existing postpartum urinary incontinence.

There were differences in the way the studies reported their results, in particular the interpretation of the intention to treat (ITT) analysis. Intention to treat analysis is 'a strategy for analyzing data in which all participants are included in the group to which they were assigned, whether or not they completed the intervention given to the group'. ${ }^{(32)}$ A number of studies claimed to conduct an ITT analysis, but had not included the participants originally randomised to the treatment and control groups and for whom no data were available at the end of the trial. To ensure consistency in the review, three versions of a meta-analysis were conducted for each group of studies using the data reported for each trial. These analyses are based on:

1. An assumption that all missing participants from the treatment and control groups did not have urinary incontinence

2. An assumption that all missing participants from the treatment and control groups did have urinary incontinence

3. The data reported in the results of the study.

All three meta-analyses are presented in the results section below.

Two studies were pooled in a meta-analysis for the effectiveness of a PFME program on exercising frequency. ${ }^{(31,33)}$ However, differences between the time of intervention, outcome measures and methodological quality meant that data from most studies were analysed in narrative summaries for the outcomes frequency of exercising and pelvic floor muscle strength.

\section{Description of studies}

Although the search for studies was not limited to English language papers, only one potentially relevant study was located in a language other than English. This Italian language article was translated, facilitating the assessment for inclusion by the primary reviewers. All studies that met the quality criteria for one or more aspects of the review were in English. The authors of these 
papers represented the fields of physiotherapy, nursing, and obstetrics and gynaecology and the trials were conducted in Australia, New Zealand, Norway, the United Kingdom, and the United States. In the initial search a number of studies were located as conference presentations. Most of these were published as full articles in journals at a later date and the final group of studies included in the review were all full-text articles.

In total, 34 studies were identified as relevant to the review and were assessed against the inclusion criteria. Twenty-two of these studies met the inclusion criteria for one or more aspects of the review. (See Appendix VIII for studies excluded in this process.) After assessment of methodological quality by the review panel, six RCTs were included in the effectiveness of PFME on urinary incontinence outcome of the review. ${ }^{(26,30,31,33-35)}$ Eleven studies were included in the review for the two other outcomes of interest as they provided data about frequency of PFME undertaken and strength of pelvic floor muscle contractions. ${ }^{(26,27,30,31,33-39)}$ The review panel excluded 11 studies due to poor methodological quality or lack of clinical relevance to health professionals' practice given the clinical practice context on which this review was focused. See Appendix IX for details of these studies. The figure below illustrates the process of assessing studies for inclusion in the review.

Figure 1. Study assessment process

\section{<insert fig_01.eps>}

The stated hypotheses and/or outcomes of the included studies focussed on:

- prevention of urinary incontinence ${ }^{(26,34,35)}$

- treatment of urinary incontinence ${ }^{(30,31,33)}$

- prevention and treatment of urinary incontinence $e^{(37,38)}$

- performance of pelvic floor muscle exercises, ${ }^{(26,31,33)}$ and

- improved pelvic floor muscle strength. ${ }^{(27,36,39)}$

\section{Types of participants}

Appendix X lists the inclusion and exclusion criteria used by the studies to select participants. Two papers included in the exercising and pelvic floor muscle strength aspects of the review reported on a continuing study. ${ }^{(37,38)}$ The second paper described data collected at a 12-month follow-up of some of the participants recruited for the research described in the first paper. The authors give no indication as to whether the participants were informed that they would be contacted at a later date to collect further data. Participants were matched in pairs for the first and second stages of the research. However, because some participants were not available at the 12month follow-up, data were collected for fewer matched pairs.

Four studies selected their samples on the basis of an existing risk. ${ }^{(26,31,33,35)}$ Women who had forceps or ventouse deliveries and/or babies $4000 \mathrm{~g}$ or more comprised the sample for one of the studies. ${ }^{(26)}$ Two studies selected women with existing urinary incontinence at three months postpartum for their samples. ${ }^{(31,33)}$ One study selected only women with bladder neck mobility (at least $5 \mathrm{~mm}$ on a linear scale), measured using a perineal ultrasound. ${ }^{(35)}$

Most of the studies included women of all parities. ${ }^{(26,27,31,33,36-38)}$ Four of the studies limited their samples to primiparas/primagravidas. ${ }^{(30,34,35,39)}$ There were no exclusions according to type of delivery for the participants in seven of the trials. ${ }^{(31,33-35,37-39)}$ Three studies $^{(26,27,36)}$ selected women who had vaginal deliveries only and one study excluded women who were delivered by elective caesareans. ${ }^{(30)}$ A number of the trials had further exclusion criteria for their samples as noted in Appendix X.

Participants were recruited at antenatal check-ups (including ultrasounds) ${ }^{(27,34-36,39)}$, in hospital within days of delivery ${ }^{(26)}$, or by letter or telephone contact after leaving hospital using the hospital records. ${ }^{(31,33)}$ In one study women were recruited in a private obstetrician's office ${ }^{(36)}$ and in two 
studies participants received money and/or services for taking part in the study ${ }_{(27,30)}$ Participants in the first study received US\$30, a free pap smear and babysitting if required. ${ }^{(27)}$ In the second study participants received approximately US\$150. ${ }^{(30)}$ It is not clear where recruitment took place for the sample in one study, but all participants had delivered at the same hospital. ${ }^{(37)}$

\section{Types of Interventions}

The interventions included antenatal and postnatal PFME programs comprising instruction sessions and other components, such as take home materials, provided once or many times by a range of health care professionals. Most of the interventions differed in the type and timing of instruction provided, the combination of components (such as feedback and reinforcement strategies), and the type and number of exercises recommended in the home program. Appendix $\mathrm{XI}$ lists the details of the interventions delivered by the studies.

The timing of the interventions was equally divided between the antenatal and postnatal periods. Antenatal PFME were examined in five studies. ${ }^{(30,34-36,39)}$ The intervention in the other five studies was a postnatal PFME program. ${ }^{(26,27,31,33,37)}$ One study provided an instruction session only once (but contacted participants each week for six weeks by telephone) ${ }^{(27)}$ and participants in one study had, with twelve occasions, the most number of occasions of instruction. ${ }^{(34)}$ One study included no information about the number of instruction sessions, but up to eight contacts with participants were possible, on the basis of the information provided. ${ }^{(36)}$

The home program of pelvic floor muscle exercising recommended for women in the treatment groups varied greatly across the studies. At the higher limits exercising the pelvic floor muscles 80 to 100 a day were recommended. ${ }^{(31,33,36,39)}$ Performing between 20 and 50 exercises daily was recommended in four studies ${ }^{(30,34,35,37)}$ and one study suggested participants should perform 25 exercises three times a week. ${ }^{(27)}$ The authors of one study do not refer to the number of exercises participants should perform except to state they should perform them three times daily. ${ }^{(26)}$

These differences are also reflected in the type of PFME that participants were instructed to perform. The participants in one study were instructed to hold each contraction for 3-6 seconds. ${ }^{(26)}$ Two studies used the same exercising program, comprising 8-10 sessions daily of 810 fast and slow contractions. ${ }^{(31,33)}$ The participants in two studies were asked to perform 8-12 contractions twice daily with each contraction being held for 6-8 seconds, followed by 3-4 fast contractions and then a 6 second rest. ${ }^{(34,37)}$ A graduated exercising program, where the number of exercises performed was increased over the period of the research, was used in three studies. ${ }^{(27,30,35)}$ One study asked participants to perform two types of exercises, one to improve strength and the other to improve endurance or maintained pressure. For pelvic muscle strength the contractions were to be held for 6 seconds and over the 6-week study period the number of exercises increased from 15 per day three times a week to 25 per day three times a week. The exercise program for the maintaining pressure aspect of the study involved increasing the length of time a contraction was held from 6 seconds at the commencement of the intervention to 12 seconds by the end of the intervention period. ${ }^{(27)}$ In the beginning of one study participants were instructed to perform twice daily, three sets of 8 contractions (held for 6 seconds) with a twominute rest between each set. At 14 weeks into the 20 -week intervention period, participants were asked to increase the number of exercises to 12 per set. ${ }^{(35)}$ Another study used a five level program called Graduated Strength Training, which was developed by two of the researchers. The number of contractions performed daily is reduced from five sets of 10 contractions daily to one/two sets of 5 contractions weekly as muscle strength improves. Improved muscle strength is achieved through gradually holding contractions for longer, starting with short contractions and building up to 10 -second contractions at high intensity. ${ }^{(30)}$ Participants were asked to perform 50 full strength short contractions twice daily in one study ${ }^{(39)}$ and another used Kegel's exercise program asking participants to perform 100 contractions per day or perform the contractions for 20 minutes three times a day, each contraction being held for 3 seconds. ${ }^{(36)}$ 
All studies included in the review had an education component in the intervention and most studies included feedback for participants when they were being taught the exercises. ${ }^{(26,27,30,31 \text {, }}$ 34-37) The feedback related to performing the PFME correctly and/or pelvic floor muscle strength and was given after digital examination or instrumental measure. Fewer studies provided takehome materials, such as brochures or written instructions ${ }^{(26,27)}$, and aids to motivate participant exercising and reinforcement. ${ }^{(26,34,36)}$ In one study participants were contacted by weekly telephone calls encouraging them to exercise. ${ }^{(27)}$

There was a range of health professionals providing the instruction sessions to women in the studies and this reflected the professional interests of the researchers involved in the studies. In five studies a physiotherapist delivered the instruction session(s). ${ }^{(26,31,34,35,37)}$ A nurse trained by a physiotherapist provided instruction in one study. ${ }^{(33)}$ Nurses also provided the instruction sessions in two studies. ${ }^{(27,30)}$ In one of the studies an obstetrician provided instruction. ${ }^{(36)}$ For all these studies the instruction was provided on a one-to-one basis on the first occasion. The oneto-one contact continued for later instruction sessions in several of the studies. ${ }^{(26,31,33,35,36)}$ Subsequent instruction sessions in two studies were provided in groups. ${ }^{(34,37)}$ No information was available about the profession of the person providing the instruction or the nature of the intervention in one study. ${ }^{(39)}$ However, a midwife initially recruited participants at an antenatal check-up visit which might indicate the instruction session was delivered by the midwife on a oneto-one basis.

Two studies included components of interventions that were excluded by the review and therefore the data of those studies were used selectively. ${ }^{(27,31)}$ One included an intervention (vaginal cones) in their research that is not common practice in the context of this review. The results from one (PFME only) of three interventions (PFME only, vaginal cones, and PFME with vaginal cones) in this study were included in the urinary incontinence outcome of interest in the review. ${ }^{(31)}$ The other compared three interventions: PFME only, PFME with an intravaginal balloon device in place, and an intravaginal balloon device in place with no PFME. The results of the first intervention only were included in the pelvic floor muscle strength outcome of the review. ${ }^{(27)}$ However, for the exercising frequency outcome examined in this review the results for all groups receiving an intervention in these studies were used because the focus of the intervention for this outcome was the type of instruction methods used in the PFME program and this was provided to all participants in the intervention group.

\section{Types of outcomes}

Data collection in relation to outcomes was linked to the timing of an intervention, for example studies of antenatal PFME interventions tended to collect data closer to delivery than postnatal PFME intervention studies. Antenatal interventions were the subject of five studies with data collection carried out at between 5 to 6 weeks postpartum, ${ }^{(36)} 2$ months and 8 months postpartum, ${ }^{(39)} 3$ months postpartum, ${ }^{(34,35)}$ and 6 months postpartum. ${ }^{(30)}$ The studies with postnatal interventions collected data at 3 months postpartum, ${ }^{(26,37)}$ between 3 to 6 months postpartum, ${ }^{(27)}$ and 12 months postpartum, ${ }^{(31,33,38)}$ the maximum period for data to be included in this review. Therefore, the data for 24 and 44 months postpartum were not used. ${ }^{(31)}$ The outcomes from the studies for data collection are discussed below.

\section{Urinary incontinence}

A number of the studies that examined the effectiveness of PFME on urinary incontinence distinguished between stress and mixed (stress and urge) urinary incontinence. Three studies referred to their outcome as mixed incontinence. ${ }^{(26,31,33)}$ One study made no distinction and used the term urinary incontinence to describe the outcome being measured. ${ }^{(34)}$ Stress urinary incontinence only was the outcome measured by two studies, ${ }^{(30,35)}$ with one of these specifically noting that data from participants reporting urge incontinence only were excluded. ${ }^{(35)}$ The definitions of urinary incontinence used by studies are listed in Appendix XII.

All studies included in this outcome of interest to the review presented their data for incontinence as dichotomous, frequently modifying scaled responses to do so. For example, in one study 
participants' responses of 'occasionally', 'often', or 'always' were all coded as urinary incontinence. ${ }^{(26)}$ In contrast, another study coded responses of 'once a week or more' as urinary incontinence and 'less than once a week' as no urinary incontinence ${ }^{(34)}$ Continuous outcomes, such as severity of urinary incontinence ${ }^{(26,33)}$ and pad tests of leakage ${ }^{(31,35)}$ were also reported but these outcomes were not examined in this review due to differences between study methods.

Three of the studies collected their data through self-reports and bladder diaries. ${ }^{(26,34,35)}$ The other three studies relied solely on self-reports to collect data. ${ }^{(30,31,33)}$

\section{Frequency of PFME exercising}

Both dichotomous and continuous data were reported in the studies included in the effectiveness of a PFME program on frequency of pelvic floor muscle exercising. Two studies used a dichotomous outcome based on the number of occasions PFME were performed each week. ${ }^{(26,}$ 37, 38) That is, a participant reporting exercising two or more times a week was coded as adequate exercising and any less exercising was coded as inadequate. ${ }^{(26)}$ The second study used performing PFME three or more times a week or less than three times a week as coding categories. $^{(37,38)}$ In two studies a dichotomous outcome was used to report participants' exercising in the previous month. ${ }^{(31,33)}$ One of these studies also reported if participants were exercising daily using a dichotomous outcome. ${ }^{(31)}$ The same studies asked participants how many PFME they were performing daily and this outcome was presented as a continuous variable. ${ }^{(31,33)}$ The results of one study could not be compared with the other studies because the outcome for the number of days PFME were performed was reported as a ratio of the number of days that PFME were recommended. ${ }^{(27)}$

Self-report was the data collection method used in four studies, ${ }^{(26,31,33,38)}$ with training diaries used to collect data in the other two studies. ${ }^{(27,37)}$

\section{Strength of pelvic floor muscles}

The strength of pelvic floor muscles was measured by four differently named instruments in the nine studies included in the outcome of interest to the review. All the studies reported their results as continuous outcomes. Vaginal balloon catheters, which measure in units of $\mathrm{cmH}_{2} \mathrm{O}$, were used three studies. ${ }^{(34,37,38)}$ Three studies used perineometers to measure pelvic muscle strength in mmHg. ${ }^{(31,35,36)}$ The same unit of measure was reported in two studies, one using a WISAP Vagino-Tonograph and the other with an intravaginal balloon device. ${ }^{(27,39)}$ An instrumented speculum was used in one study to measure pelvic muscle strength presenting results in newtons (a unit of force). ${ }^{(30)}$

\section{Methodological quality of included studies}

Full details of all studies included in the review are at Appendix XVIII.

Appendix XIII outlines the main methodological quality characteristics of the studies in the review. For inclusion in the incontinence outcome of interest in the review, an assessment tool based on the methodological quality criteria of randomised controlled trials was used. The quality assessment of studies for the exercising frequency and pelvic floor muscle strength outcomes was performed using criteria that allowed for non-randomised controlled trials to be included. Six RCTs were included in the urinary incontinence outcome of review and some of these were also included in the other outcomes of interest to the review. ${ }^{(26,30,31,33-35)}$ A further five studies were included in these other outcomes of interest. ${ }^{(27,36-39)}$

Power calculations were performed to determine the sample size in five studies, ${ }^{(26,31,33,34,36)}$ although one study based calculations on a previous study's results. ${ }^{(35)}$ Two studies $^{(26,33)}$ recruited large sample sizes: one had with 370 women in their treatment group and 350 women in 
their control group; ${ }^{(26)}$ the other randomised 371 to their treatment group and 376 women to their treatment group. ${ }^{(33)}$ Fewer participants were assigned to treatment and control groups in two studies $^{(34,35)}$ with 148 and 139 women in the treatment groups and 153 and 129 in the control groups, respectively. One study initially recruited 198 women to comprise their 99 matched pairs $^{(37)}$ and another assigned 39 women to the treatment group relevant to this review and 117 women to their control group. ${ }^{(31)}$ The remaining studies recruited less than 50 women to each of their groups. $27,30,36,39)$

As required, randomisation of participants to treatment or control groups occurred in the six studies $^{(26,30,31,33-35)}$ included in the first aspect of the review, as well as a further study. ${ }^{(27)}$ Randomisation was conducted using a variety of methods. Four studies carried out relatively sophisticated randomisation techniques using third parties, assignment with blank envelopes, and stratified randomisation using a computer program. ${ }^{26,31,33,34)}$ Two studies used random number generation to assign participants to the groups. ${ }^{(30,35)}$

Of the remaining studies included in the second aspect of the review, one provided little information on which to assess the randomisation process, ${ }^{(27)}$ another selected a convenience sample from women attending an obstetrician's office for antenatal and postnatal visits, ${ }^{(36)}$ participants were assigned to the treatment or control group by matching pairs, ${ }^{(37,38)}$ and the researchers assigned women to the two groups in an alternate sequence as they presented for an antenatal check-up by a midwife. ${ }^{(39)}$

All studies reported women in the control groups received 'usual' or 'customary care' and were not discouraged from performing PFME, but due to the nature of the intervention, participants in the studies could not be blinded to their allocation. Performance bias was addressed in most studies by ensuring that the same individual delivered the intervention to all participants in the treatment groups. ${ }^{(27,30,31,34-37)}$ One study used taped instructions to provide consistency in delivering the intervention. ${ }^{(27)}$ In the study conducted across three sites and a standard set of diagrams and instructions was used to reduce performance bias. ${ }^{(33)}$ The interventions in two studies were delivered by three and five (respectively) physiotherapists. ${ }^{(26,34)}$ One study provided no information on which to assess performance bias. ${ }^{(39)}$

In most of the studies detection bias was avoided by blinding the data collectors (eg. those taking perineometer readings in Wilson and Herbison's study) or data assessors to the group allocation of participants. ${ }^{(26,30,31,33-35)}$ There was no attempt to reduce detection bias in one study ${ }^{(36)}$ and no information was provided in four studies to determine whether detection bias may have affected the studies' results. ${ }^{(27,37-39)}$ These latter studies were part of the narrative comparisons related to objectives 3 and 4 of the review.

Less than $10 \%$ of participants in the treatment and control groups were unavailable for data collection in three studies. ${ }^{(26,27,34)}$ Two studies reported less than $20 \%$ of participants in both groups were lost at data collection. ${ }^{(30,35)}$ No significant differences between the groups were found in one of these studies. ${ }^{(35)}$ The other reported a higher number of women in the treatment group had delivered by caesarean, but believed this difference had not affected results. ${ }^{(30)}$ At the 2-month postpartum data collection point, reported $16 \%$ of the participants in the treatment group and $33 \%$ of the control group were not available for data collection in one study. ${ }^{(39)}$ These researchers provide no information about participants lost to follow-up. Between $20-30 \%$ of the treatment groups in two studies were not available at data collection. ${ }^{33,36)}$ Researchers involved in one study conducted an analysis to determine differences between participants who responded to the questionnaire and non-responders. Non-responders tended to have more severe incontinence at baseline, but at one site only. ${ }^{(33)}$ Another study excluded $15 \%$ of participants at data collection if they had caesarean deliveries to ensure the groups remained comparable for that variable. ${ }^{(36)}$ Over $50 \%$ of participants dropped out of the treatment groups in two studies. ${ }^{(31,39)}$ One of these ${ }^{(39)}$ reported a very high number of dropouts from the control group also (66\%) but the other lost only $22 \%$ of the participants in their control group at data collection. ${ }^{(31)}$ The analysis for participants lost to follow-up this study was performed for the three intervention groups 
together and showed that these women were slightly younger and smoked more. Information about the women lost to follow-up in the PFME group only was not reported, but the authors noted the given reasons for withdrawal as 'lack of time and inconvenience involved with the hospital-based regimen'. In one study, reported in two papers, $18 \%$ percent of the participants from the earlier study were not included in the follow-up, however this was, for the most part, due to the study's design of matching pairs as a sample. ${ }^{(37,38)}$ Six studies reported performing intention to treat analysis (ITT) to determine the effect of participants lost to follow-up. ${ }^{(26,30,31,33-35)}$ However, as noted above, there were a number of methods used to calculate ITT. That is, some studies included the number of participants at group assignment in the calculation while others used the number of participants available at data collection to perform the calculation.

\section{Results}

\section{Effectiveness of PFME on urinary incontinence following childbirth}

The RCTs included in this part of the review have been pooled according to whether the intervention was provided in the antenatal or postnatal period. Subgroup analysis has also been performed for the two studies of postnatal PFME programs for women with existing urinary incontinence. ${ }^{(31,33)}$

\section{Antenatal PFME versus usual care}

Three studies examined whether an antenatal PFME program was more effective than usual antenatal care for preventing and/or treating postpartum urinary incontinence. ${ }^{(30,34,35)}$ In all three studies the outcomes were measured in primigravidas. The two larger studies reported significant differences between their treatment and control groups' results. ${ }^{(34,35)}$ One study's results indicated participants in the treatment group had significantly less urinary incontinence than those in the control group at three months postpartum $(p=.018$, NNT 1 in 8$) .{ }^{(34)}$ In another fewer women in the treatment group had stress incontinence than women in the control group at three months postpartum $(p=.023){ }^{(35)}$ Although the results in one study were not statistically significant, the authors note that there was less urinary incontinence in the treatment group than the control group at six months postpartum. ${ }^{(30)}$ Appendix XIV provides details of these studies.

The meta-analysis carried out on the three studies included a test for heterogeneity as well as a $p$ value to indicate whether the results are statistically significant. These calculations are reported in the lower part of each diagram. When viewing the meta-analysis of the three studies, in Figures 2 - 4 below, it is important to note a potentially important difference in that the women recruited to one study had pre-existing mobile bladder necks. ${ }^{(35)}$ However, as the other studies did not test for bladder neck mobility it could be assumed that a number of their participants would also have mobile bladder necks. Furthermore, the results from the study that included women with preexisting bladder neck mobility show that there were no significant differences in bladder neck mobility between the treatment and control groups over the course of the trial. This finding, combined with the statistically significant results for the treatment group, suggests that bladder neck mobility may not be a confounding factor in the trial. The three trials also differed in the number of occasions their intervention was delivered as outlined in Appendices $L$ and $P$. Two studies collected data about stress incontinence as an outcome ${ }^{(30,35)}$ while the third did not differentiate between types of incontinence. ${ }^{(34)}$ Data collection points were three months postpartum for two studies ${ }^{(34,35)}$ and six months postpartum for the other. ${ }^{(30)}$

Figure 2 The Effectiveness of Antenatal PFME on Urinary Incontinence Following Childbirth: Assuming All Missing Participants are not Incontinent

<insert fig_02.eps> 
Figure 3 The Effectiveness of Antenatal PFME on Urinary Incontinence Following Childbirth: Assuming All Missing Participants are Incontinent

<insert fig_03.eps>

Figure 4 The Effectiveness of Antenatal PFME on Urinary Incontinence Following Childbirth: Study Data

<insert fig_04.eps>

Figures 2 - 4 above show that results of the heterogeneity tests indicate the studies can be pooled and show a $p$ value ranging between $.002-.001$ for overall effect. Results of the pooled studies, regardless of how the calculations were performed for missing participants, indicate an intervention of an antenatal PFME program is effective in preventing and/or treating urinary incontinence after childbirth.

\section{Postnatal PFME versus usual care}

Appendix XV provides the details of the three studies that examined whether a postnatal PFME program was more effective than usual postnatal care for preventing and/or treating urinary incontinence after childbirth. ${ }^{(26,31,33)}$ One study reported urinary incontinence in their treatment group at 3 months postpartum was significantly less than in their control group $(p=.044)$ with greater difference shown $(p=.01)$ when the researchers accounted for the confounding factors of urinary incontinence prior to childbirth and continence status after delivery. ${ }^{(26)}$ Significantly fewer $(p=.037)$ women in the treatment group of one study had urinary incontinence at 12 months postpartum than women in the control group. ${ }^{(33)}$ The third study does not provide calculations for statistical significance for the PFME intervention group alone, but report that urinary incontinence was significantly less $(p=.003)$ in the combined intervention group (comprising three sub-groups of PFME, cones, and PFME and cones together) than the control group. The authors go on to note "pfme and cones appeared equally effective in treating urinary incontinence". ${ }^{(31)}$ The figures used in the meta-analysis below are those given for the PFME intervention group only.

Figure 5 The Effectiveness of Postnatal PFME on Urinary Incontinence Following Childbirth: Assuming All Missing Participants are not Incontinent

\section{<insert fig_05.eps>}

Figure 6 The Effectiveness of Postnatal PFME on Urinary Incontinence Following Childbirth: Assuming All Missing Participants are Incontinent

\section{<insert fig_06.eps>}

Figure 7 The Effectiveness of Postnatal PFME on Urinary Incontinence Following Childbirth: Study Data

\section{<insert fig_07.eps>}

In Figures $5-7$ above heterogeneity tests indicated the studies could be pooled and present statistically significant $p$ value for overall effect ranging from .02 to .009 . These results show that postnatal PFME are effective in preventing and treating urinary incontinence following childbirth. However, the differences between the three analyses in the figures suggest that the way in which participants lost to follow-up are treated in two studies affect the findings of the meta-analysis. ${ }^{(31,}$ 
These two studies had a high number of participants lost to follow-up (>20\%) and also studies recruited women with pre-existing urinary incontinence only. Despite the number of participants lost to follow-up there were other aspects of these studies that warranted their inclusion in the review. Although women with pre-existing urinary incontinence were not excluded from the third study, their sample was recruited using inclusion criteria of women who had assisted deliveries and/or large babies $(>4000 \mathrm{~g}) .{ }^{(26)}$ This study also had an earlier data collection point - three months postpartum as opposed to 12 months postpartum. A subgroup analysis was performed for the two studies with greater similarities (Figures 8-10). ${ }^{(31,33)}$ Another meta-analysis was conducted, not presented in this report, excluding one of the studies because of their high number of participants lost to follow-up, but no significant differences were evident in the analysis. $^{(31)}$

The following figures present the meta-analysis of the two studies in the subgroup of postnatal PFME programs for women with pre-existing urinary incontinence. ${ }^{(31,33)}$

Figure 8 Subgroup analysis: Pre-existing Urinary Incontinence. The Effectiveness of Postnatal PFME on Urinary Incontinence Following Childbirth: Assuming All Missing Participants are not Incontinent

\section{<insert fig_08.eps>}

Figure 9 Subgroup analysis: Pre-existing Urinary Incontinence. The Effectiveness of Postnatal PFME on Urinary Incontinence Following Childbirth: Assuming All Missing Participants are Incontinent

\section{<insert fig_09.eps>}

Figure 10 Subgroup analysis: Pre-existing Urinary Incontinence. The Effectiveness of Postnatal PFME on Urinary Incontinence Following Childbirth: Study Data

\section{<insert fig_10.eps>}

Because of the large number of women lost to follow-up in these studies, the analyses presented in Figures 8 - 10 above show great variation depending upon how the missing data are treated. However, the overall effect is statistically significant for the analyses in Figure 9 (all missing participants are incontinent) and Figure 10 (study data) are similar ( $p=.001$ and $p=.005)$ and indicate that a postnatal PFME program for women with existing urinary incontinence after childbirth is effective in treating that incontinence.

\section{Effectiveness of a PFME program on frequency of exercising}

The focus of this part of the review is on the different components of a PFME program. Of particular interest was the number of instruction sessions, whether feedback was provided to participants about the effectiveness of their pelvic floor muscle contractions, if information materials were provided, and the use of adherence aids such as exercise diaries and posters. All programs included an exercising program to perform at home.

Five studies examined the frequency of exercising as an outcome of a PFME program. ${ }^{(26,27,31,33,}$ ${ }^{37,38)}$ Four of the studies were RCTs and the other a non-RCT reported in two papers. Due to the different outcomes measured by the studies and the way in which outcomes were measured, most are discussed in a narrative summary below. All studies included in this aspect of the review 
had tested postnatal interventions that included, at least, an initial instruction session and a home exercise program. The outcomes measured PFME undertaken in the previous month, week, and daily, the number of PFME undertaken daily, and exercising as a ratio of the prescribed amount of exercising.

Two studies could be pooled in a meta-analysis for the effectiveness of a postnatal PFME program on exercising frequency. ${ }^{(31,33)}$ Appendix XVI lists the relevant details of all studies included in this outcome of interest to the review.

\section{Three individual instruction sessions and home training program / Four individual instruction sessions, instrument feedback and home training program versus usual postnatal care}

The interventions of two studies are presented together because of the similarities between them. ${ }^{(31,33)}$ The main difference between the studies is that one study did not include feedback about the effectiveness of PFME to participants as part of their intervention. ${ }^{(33)}$

The data collected by these studies included the number of participants who had performed PFME in the previous month and how many exercises participants were performing each day. One of the studies also asked participants if they were performing PFME daily. ${ }^{(31)}$

\section{Performed PFME in Previous Month}

The studies were pooled for the analysis of this outcome. However, the findings should be considered with caution due to the high number of participants lost to follow-up, over $20 \%$ in both studies, and the addition of feedback about the strength of their pelvic floor muscle contractions to participants in the treatment group in one study. ${ }^{(31)}$

This study also delivered three different physical interventions but all women receiving an intervention were given the same care in terms of the components of the PFME program important in this part of the review. Therefore, the results used in the meta-analysis are those for the combined intervention group. At the 12-month postpartum data collection point participants were categorised as performing PFME in the previous month or not performing PFME in the previous month.

Because performing PFME in the previous month is a positive outcome (in contrast to the analyses above for urinary incontinence which is a negative outcome) the meta-analyses Figures 11 and 12 below show treatment on the right hand side of the graph.

Figure 11 The Effectiveness of a PFME Program on Frequency of Exercising in Previous Month: Assuming all Missing Participants are Exercising

\section{<insert fig_11.eps>}

Figure 12 The Effectiveness of a PFME Program on Frequency of Exercising in Previous Month: Study Data

\section{<insert fig_12.eps>}

An analysis based on the assumption that 'all missing participants are not exercising' was not presented here because the heterogeneity test indicated that the studies' results in this form should not be pooled. Individually, the studies reported their treatment group exercised in the previous month in statistically significantly higher numbers than their control group $\left(p<.001 ;{ }^{(33)}\right.$ $p=.07^{(31)}$ ). These findings are reflected in the pooled analyses, showing that an education and instruction program in PFME is effective in increasing exercising frequency (overall effect $p=.00001)$. 
Number of PFME Performed Daily

In one study participants in the treatment group were performing, on average, a significantly higher number of PFME daily (mean=20) than participants in the control group (mean=15, $p<.001$ ) at 12 months postpartum. ${ }^{(33)}$ The other study also collected data about how many PFME women were performing daily. The researchers found women in the three intervention groups were performing a significantly higher number of PFME daily (mean=59) than women in the control group (mean $=35, p<.0005)$ at 12 months postpartum. ${ }^{(31)}$

In respect to this outcome, these studies used different outcome measures and therefore the results could not be pooled in a meta-analysis. One study measured the number of PFME performed daily with means and standard deviation ${ }^{(33)}$ and the other reported the same outcome with means and range. ${ }^{(31)}$

Performed PFME Daily

Only one study asked participants if they performed PFME daily. ${ }^{(31)}$ At the 12 -month postpartum data collection point a significantly higher number of women in the three intervention groups (48\%) performed PFME daily compared with women in the control group $(9 \%, p<.0005)$.

Two individual instruction sessions, home training program, information booklet, and adherence aids versus usual postnatal care

Performed $P F M E=>$ Twice/Three Times Weekly

The intervention in one study included extensive take home materials as adherence aids for women in the treatment group. Data were collected at three months postpartum and a significantly higher $(p=.001)$ number of women in the treatment group $(83.9 \%)$ exercised twice or more each week compared with the control group (57.6\%). ${ }^{(26)}$

Eight individual instruction sessions, instrument feedback, home training program, weekly group program and training diary versus usual postnatal care

Performed PFME => Twice/Three Times Weekly

The women recruited in one study were asked about urinary incontinence and PFME exercising, plus pelvic muscle floor strength was measured, prior to the intervention (at eight weeks postpartum) and after the intervention at 16 weeks postpartum. More women in the control group than the treatment group had exercised prior to the intervention, however at the 16 week data collection point all women in the treatment group (100\%) were exercising at least three times a week compared with two-thirds (66\%) of the control group women. The study reports these results with a $p$ value of $.0000 .^{(37)}$

In a follow-up study of the participants from the 1997 study twelve months after delivery with a slightly smaller sample of 81 matched pairs. These women were asked about the frequency with which they had performed PFME since the 16 week postpartum data collection point. During this period, more women (53\%) from the original treatment group compared with women from the control group (30\%) had exercised three or more times each week (with a $p$ value calculated at $.002) \cdot{ }^{(38)}$

The studies that measured the number of times PFME were performed each week reported significant results for their treatment group's exercising frequency compared with the control group. $^{(26,37,38)}$

One individual instruction session with instrument feedback, home training program, taperecorded instructions, weekly contact and exercising diaries

Adherence ratio for performing PFME

One study randomised women into three groups. After 6 weeks of the program data were collected about how often the participants were exercising. The data were converted into an adherence ratio by dividing the number of days exercised by the number of days exercises were prescribed multiplied by 100. Data were not available for each group, but overall a very high 
adherence ratio (96.2\%) was reported across the groups. Because data were not collected for women who received usual care and were not part of this study, no conclusions can be drawn about the effectiveness of the program. ${ }^{(27)}$

Although most of the studies that examined the effectiveness of a PFME program on exercising frequency differ in a number of ways, they repeatedly show significant results that indicate a structured multi-faceted educational program can be effective compared with usual care.

\section{Effectiveness of a PFME program on pelvic floor muscle strength}

To analyse if a PFME program is effective in improving pelvic floor muscle strength, this review focussed on the components of a PFME program, including the number of instruction sessions, whether feedback was provided to participants about the effectiveness of their pelvic floor muscle contractions, if information materials were provided, the use of adherence aids, and the home exercising program recommended.

Nine studies, five RCTs and four non-RCTs, looked at changes in pelvic floor muscle strength as a result of a PFME program..$^{(27,30,31,34-39)}$ Five of these studies provided their PFME program in the antenatal period and collected data between six weeks postpartum and eight months postpartum. ${ }^{(30,34-36,39)}$ The other studies introduced their PFME program in the postnatal period and collected data between three months and 12 months postpartum. ${ }^{(27,31,37,38)}$ As noted above, measures of pelvic muscle strength were taken with a variety of instruments using different units of strength. Also, a range of statistical calculations was performed on data leading to results that are not directly comparable. For these reasons the studies are discussed in a narrative summary, with antenatal programs distinguished from postnatal programs. This distinction is made because the antenatal programs tend to focus on prevention, while the postnatal programs are concerned with treatment and restoration of muscles in the pelvic floor after childbirth. Appendix XVII lists the relevant details of all studies included in this aspect of the review.

\section{Antenatal PFME programs versus usual care}

Three studies reported statistically significant differences in pelvic floor muscle strength between the treatment groups and control groups, suggesting that an antenatal PFME program is effective in this respect compared to usual care. ${ }^{(34,36,39)}$ The other two studies reported no significant differences in pelvic floor muscle strength between the two groups. ${ }^{(30,35)}$

Two studies provided an antenatal PFME program on multiple occasions from 20 week gestation to delivery. ${ }^{(34,35)}$ At the two data collection points (36 weeks gestation and three months postpartum) women in the treatment group in one study had significantly higher pelvic floor muscle strength than women in the control group (Mann-Whitney $U$ test: 36 weeks gestation $p=.008 ; 3$ months postpartum - $p=.048){ }^{(34)}$ The antenatal PFME program in the other RCT found there was no difference in the pelvic floor muscle strength between the two groups at three months postpartum. ${ }^{(35)}$

One study provided a PFME program on a one-to-one basis to women in the treatment group at 20 weeks gestation. At six months postpartum there were no significant differences between the pelvic floor muscle strength of women in the treatment and control groups. ${ }^{(30)}$

It is unclear how many times the treatment group received an antenatal PFME program in two studies. ${ }^{(36,39)}$ The author of one study describes one occasion at between 32 and 36 weeks gestation, where women in the treatment group were instructed in pelvic floor anatomy and the benefit of PFME. A perineometer reading of pelvic muscle strength was provided to women at every visit until delivery. At the postpartum visit, between 5-6 weeks, women in the treatment group had significantly higher pelvic floor muscle strength than women in the control group 
$(p<.01) .{ }^{(36)}$ Women in the other study had their pelvic floor muscle strength measured at baseline, $34,37,38$, and 39 weeks gestation and at two and eight months postpartum using a perineometer (WISAP Vagino-Tonograph 4001). There was greater improvement in pelvic floor muscle strength of the treatment group at 39 weeks gestation, although not statistically significant. The two-month data collection point showed the pelvic floor muscle strength of the treatment group $(42.9 \mathrm{mmHg}$ ) was significantly better than the control group $(34.1 \mathrm{mmHg}$, $p<.05){ }^{(39)}$

\section{Postnatal PFME programs versus usual care}

Like the antenatal PFME programs, some studies found significant differences in pelvic floor mi)

Participants in one study showed no statistically significant differences in the average change in either the 'maximum pressure' measure of strength or the 'pressure over time' measure of strength between the three groups in the study, but the change in strength was higher for the exercising groups compared with the control group. ${ }^{(27)}$

The women in another study showed a significant median improvement in the treatment group (5.3 $\mathrm{cmH} 2 \mathrm{O}$ ) compared with the control group $(0.8 \mathrm{cmH} 2 \mathrm{O}, p<.01) .{ }^{(37)}$ In the follow-up study twelve months after delivery, the pelvic floor muscle strength of the smaller sample of 81 matched pairs was measured. There had been no contact between the researchers and participating women in the interim. The average improvement in the strength of pelvic floor muscles of the treatment group $(4.4 \mathrm{cmH} 2 \mathrm{O})$ was still significantly better than the average change in strength of the control group $(1.7 \mathrm{cmH} 2 \mathrm{O}, p<.001) .{ }^{(38)}$

In the study in which three intervention groups were examined, two of which used vaginal cones as part of the intervention. Because the use of vaginal cones was excluded as an intervention in the review the data available for the exercising group only are discussed in this section of the review. While the treatment group had a higher measure of pelvic floor strength (maximum 13.6 $\mathrm{cm} \mathrm{H} 2 \mathrm{O}$, sustained $7.9 \mathrm{cmH} 2 \mathrm{O}$ ) than the control group (maximum $13.1 \mathrm{cmH} 2 \mathrm{O}$, sustained 6.7 $\mathrm{cmH} 2 \mathrm{O}$ ) at 12 months postpartum, calculations were not reported for statistical difference between these two groups. Overall, there were no significant differences in pelvic floor muscle strength between the intervention groups together and the control group. ${ }^{(31)}$

\section{Discussion}

The outcomes of interest in this review were:

- Non-occurrence of urinary incontinence following childbirth

- A change in the frequency, duration, or severity (as appropriate) of urinary incontinence up to twelve months following childbirth.

- A change in the strength of pelvic floor muscle contractions

- Period of time PFME continued after initial instruction

- Frequency of PFME undertaken

- Women's awareness of the importance of PFME

- Satisfaction with PFME instruction.

For the first two outcomes, results were available only for non-occurrence or resolution of urinary incontinence experienced by women following childbirth. This was a dichotomous outcome with participants reported as continent or incontinent in all studies included in the review. As noted earlier, in all six studies this dichotomous outcome was measured by self-report. Results for severity and length of time urinary incontinence was experienced were either not reported in comparable measures or not an outcome examined by the studies. 
In relation to the other outcomes, which focused on the components of a PFME program that might be effective, data were available to the review for:

- Improved strength of pelvic floor muscle contractions

- Frequency of PFME undertaken

The studies included in the review had not examined the exact period of time PFME were continued after initial instruction. No studies were found that addressed women's awareness of importance of PFME or satisfaction with instruction.

\section{Effectiveness of antenatal and postnatal PFME in preventing or resolving urinary incontinence}

Six RCTs met the inclusion criteria for the primary objective of the systematic review. The results of the review indicate that antenatal PFME and postnatal PFME are effective in reducing or resolving urinary incontinence following childbirth. A subgroup analysis showed that postnatal PFME also have a significant effect on reducing or resolving urinary incontinence in women with existing postpartum urinary incontinence. Some women in one of the studies had experienced urinary incontinence during their pregnancy. ${ }^{(33)}$ This aspect of the review's findings are important in that they relate to recently published research that has shown that urinary incontinence following childbirth is strongly associated with urinary incontinence before childbirth and during pregnancy. ${ }^{(8,40)}$ In particular, it has been reported that urinary incontinence in pregnancy and the postpartum period followed a general pattern of increased urinary incontinence during pregnancy and resolution of urinary incontinence in the postpartum period, particularly in the first six weeks after delivery. These authors suggest that the proportion of women with urinary incontinence at six weeks postpartum was approximately the same at 12 months after delivery. ${ }^{(40)}$ Another study found that two-thirds of women with antenatal stress urinary incontinence were incontinent 15 years later. ${ }^{(7)}$ This review shows that PFME can decrease the number of women with urinary incontinence after childbirth, which for some women will resolve short term urinary incontinence and for others may reduce the likelihood of urinary incontinence much later in their life.

\section{Effectiveness of a PFME program in encouraging pelvic floor muscle exercising frequency and adherence}

Four RCTs and two non-RCTs met the inclusion criteria for this objective in the review. Linking the frequency of performing PFME with the incidence of urinary incontinence, Glazener ${ }^{(33)}$ reported that the women in their intervention group who were performing PFME in the previous month and performing more PFME were significantly less likely to have any incontinence. Reilly's study ${ }^{(35)}$ reported similar findings, stating that women performing PFME for at least 28 days were less likely to have postpartum stress urinary incontinence compared to women performing PFME for less than 28 days.

The results of this review support previous study findings that there is little evidence to suggest that a high-intensity PFME program is more effective than a low intensity PFME regimen of exercising. ${ }^{(18)}$ In this study a high-intensity program involved frequent instruction sessions (24 in total) over an extended period of time (six months) as well as a self-directed program at home, while a low-intensity program referred to only one individual instruction session then self-directed home exercises. However, the participants in one study were seen on one occasion only, ${ }^{\left({ }^{30}\right)}$ compared with twelve, five, four, three and two occasions respectively ${ }^{(26,31,33-35)}$ and was the only trial that didn't report significant differences in urinary incontinence between the treatment and control groups. This suggests that, although a large number of individual sessions are not required, more than one may improve the effectiveness of a PFME programme.

No conclusions about the effectiveness of feedback to the individual about pelvic floor muscle strength, for example perineometer measures, as part of a PFME program can be reached in this review due to the limited information provided in the studies. Some form of feedback was included in most of the studies, the exception being Glazener's study ${ }^{(33)}$ in which feedback was not discussed.

Frequency of exercising as a result of a PFME program was significantly improved, even when 
participants had no contact with researchers for eight months, as in Morkved and Bo's study of 1997 and follow-up in 2000. ${ }^{(37,38)}$ Differences between the PFME programs, such as number of times an instruction session was provided, the content of the program, and the time elapsed between the intervention and data collection make it difficult to determine precisely the aspects of a PFME program that might improve exercising frequency. However, the frequency with which women should perform PFME is suggested by some researchers, who describe 'adequate frequency' as 'at least every second day'. ${ }^{(41)}$ From the findings of these studies, it might be inferred that any PFME program will improve the frequency with which women perform PFME.

\section{Effectiveness of a PFME program on improving pelvic floor muscle strength}

Five RCTs and four non-RCTs met the inclusion criteria for this objective in the review. The mixed results mean that no conclusions can be reached about the effectiveness of a PFME program, antenatal or postnatal, on pelvic floor muscle strength. However, the authors of one study noted that the postpartum pelvic muscle strength of women in the treatment group appeared to return earlier than women in the control group. ${ }^{(30)}$ When the type of training exercise program (for example fast and slow contractions or graduated exercise programs) and the number of exercises recommended are examined against the pelvic floor muscle strength findings of the studies, there appears to be an association. The studies with more complicated programs, that is those involving a graduated training program, did not find significant improvement in pelvic floor muscle strength for their treatment groups. ${ }^{(27,30,35)}$ However when the other outcomes, urinary incontinence and frequency of exercising, were examined with the type of training program, results were mixed.

\section{Reviewers' conclusions}

In terms of the effectiveness of PFME programs, the results of this review indicate that urinary incontinence following childbirth can be improved by performing PFME and that any form of a specific PFME program appears to improve exercising frequency. However, the value of individual components of PFME programs, such as take home materials, reminder telephone calls, and feedback of exercising effectiveness are less clear. An interesting finding by Chiarelli, Murphy \& Cockburn when they examined the acceptability of their PFME program and reasons for non-completion was that women found the information booklet helpful, but reported that they would have found it less helpful if they had not received information and advice from the therapist directly. ${ }^{(41)}$ Some of these authors, in a separate paper, also noted that the current practice of encouraging women to return home as soon as possible after delivery resulted in these women being lost from their study. ${ }^{(26)}$ This practice has wider implications. Women who spend little time in hospital after delivery, unless part of a home visiting program, have less contact with health professionals in the postpartum period and therefore less opportunity to obtain further advice and encouragement to perform PFME.

It is reasonable to conclude that contact with the health professionals providing the instruction and advice, either in the form of weekly telephone calls or monthly group sessions, affect the frequency with which women performed PFME and consequently the better urinary continence outcomes. Women in the control groups of the studies in the review received 'usual care', which in some cases included instruction in performing PFME and in others a standard information package about postnatal care given to all women leaving hospital after childbirth. This review supports the findings of another study in which the authors suggest that written information alone, provided to women postpartum, may not be adequate to encourage subsequent PFM exercising. ${ }^{(41)}$

Although the review did not identify the specific number of occasions a PFME program needs to be provided to have a significant effect on urinary incontinence, at least two instruction sessions are suggested by the findings. Reinforcement by means of personal contact of the importance of performing PFME is emphasised in most of the studies included in this review. A number of studies also note that advice about how to fit PFME into a busy mother's day is important. The 
authors of one of these studies suggest that women should perform PFME 'at least every second day ${ }^{(41)}$ which is supported by Morkved and $\mathrm{Bo}^{(42)}$ who note that muscle strength is maintained with two training sessions each week. Pelvic floor muscle programs that involve a number of contacts with women in the postpartum period not only provide reinforcement of the message but also the occasion for women to perform PFME and therefore improve their chances of resolving or reducing urinary incontinence.

A number of studies reported a high percentage of women lost to follow-up and the data collected in most of the studies relied on self-reports relating to urinary incontinence and/or frequency of exercising. These factors may have affected the overall results of the review. However wherever possible, tests for heterogeneity were carried out to determine if studies should be combined in meta-analyses and in other cases the results' limitations are acknowledged.

\section{Implications for practice}

- Encourage women to undertake both antenatal and postnatal PFME (E1).

- Pay particular attention to women with antenatal and postnatal urinary incontinence in providing advice and PFME instruction (E1).

- To encourage adherence and continuation, PFME education programs should be multifaceted with a number of components, rather than only supplying an information booklet (E4).

- Include PFME as a specific program in all antenatal and postnatal care, incorporating at least two individual instructions sessions into the program (E1).

- Provide postpartum contact, particularly for those discharged early, either by telephone, electronic or home visits (E4).

- Design PFM home exercise programs that are realistic given the demands on a mother and that can be incorporated into her daily routine in terms of number and frequency. Two or more training sessions per week are recommended (E4).

- Health professionals working with women in the postpartum period should ask about symptoms of incontinence to ensure assistance is offered to those experiencing urinary incontinence (E4).

Implications for research

More research is needed on the following:

- the educational components of a PFME program that improve the frequency of PFM exercising.

- the effectiveness of antenatal PFME for treating antenatal or postnatal urinary incontinence.

- the effectiveness of postnatal PFME for all women in the postpartum period, not just those with existing urinary incontinence or with other potential risk factors.

\section{Potential conflict of interest}

There are no conflict of interest issues for the authors and review panel of this systematic review.

\section{References}

1. Abrams $\mathrm{P}$, Cardozo L, Fall M, Griffiths D, Rosier P, Ulmsten U, et al. The Standardisation of Terminology in Lower Urinary Tract Function: Report from the Standardisation Subcommittee of the International Continence Society; 2001. 
2. Chaliha C, Bland JM, Monga A, Stanton SL, Sultan AH. Pregnancy and delivery: a urodynamic viewpoint. British Journal of Obstetrics \& Gynaecology 2000;107(11):13541359.

3. Groutz A, Gordon D, Wolman I, Jaffa A, Kupferminc MJ, Lessing JB. Persistent postpartum urinary retention in contemporary obstetric practice: definition, prevalence and clinical implications. Journal of Reproductive Medicine 2001;46(1):44-8.

4. Peyrat L, Haillot O, Bruyere F, Boutin JM, Bertrand P, Lanson Y. Prevalence and risk factors of urinary incontinence in young and middle-aged women. BJU International 2002;89(1):61-66.

5. Sampselle CM. Behavioral intervention for urinary incontinence in women: evidence for practice. Journal of Midwifery and Women's Health 2000;45(2):94-103.

6. Dimpfl TH, Hesse $U$, Schusser B. Incidence and cause of postpartum urinary stress incontinence. European Journal of Obstetrics and Gynaecology 1992;43:29-33.

7. Dolan LM, Hosker GL, Mallett VT, Allen RE, Smith ARB. Stress incontinence and pelvic floor neurophysiology 15 years after the first delivery. BJOG: an International Journal of Obstetrics and Gynaecology 2003;110:1107-1114.

8. Hvidman L, Foldspang A, Mommsen S, Bugge Nielsen J. Postpartum urinary incontinence. Acta Obstetricia et Gynecologica Scandinavica 2003;82(6):556-563.

9. MacArthur C, Lewis M, Bick D. Stress incontinence after childbirth. British Journal of Midwifery 1993;1:207-215.

10. Mason L, Glenn S, Walton I, Appleton C. The prevalence of stress incontinence during pregnancy and following delivery. Midwifery 1999;15(2):120-128.

11. Morkved S, Bo K. Prevalence of urinary incontinence during pregnancy and postpartum. International Urogynecology Journal and Pelvic Floor Dysfunction 1999;10(6):394-398.

12. Viktrup L, Lose MD, Rolff M. The symptoms of stress incontinence caused by pregnancy or delivery in primiparas. Obstetrics and Gynecology 1992;79(6):945-949.

13. Wilson PD, Herbison RM, Herbison GP. Obstetric practice and the prevalence of urinary incontinence three months after delivery. British Journal of Obstetrics \& Gynaecology 1996;103(2):154-161.

14. Peeker I, Peeker R. Early diagnosis and treatment of genuine stress urinary incontinence in women after pregnancy: Midwives as detectives. Journal of Midwifery and Women's Health 2003;48(1):60-66.

15. Kegel $\mathrm{AH}$. Progressive resistance exercise in the functional restoration of the preineal muscles. American Journal of Obstetrics and Gynecology 1948;56(2):238-248.

16. de Kruif $Y$, van Wegen P. Pelvic Floor Muscle Exercise Therapy with Myofeedback for Women with Stress Urinary Incontinence: A Meta-analysis: NHS Centre for Reviews and Dissemination; 1996. Report No.: 122687.

17. Berghmans LC, Hendriks HJ, Bo K, Hay-Smith EJ, de Bie RA, van Waalwijk van Doorn ES. Conservative treatment of stress urinary incontinence in women: a systematic review of randomized clinical trials. Br J Urol 1998;82(2):181-91.

18. Bo K, Hagen RH, Kvarstein B, Jorgensen J, Larsen S. Pelvic floor muscle exercise for the treatment of female stress urinary incontinence: III. Effects of two different degrees of pelvic floor muscle exercises. Neurourology and Urodynamics 1990;9(5):489-502.

19. Hay-Smith E, Berghmans L, Hendriks H, de Bie R, van Waalwijk van Doorn E. Pelvic Floor Muscle Training for Urinary Incontinence in Women: NHS Centre for Reviews and Dissemination; 2001. Report No.: 142392.

20. Oxman A. The Cochrane Collaboration Handbook. Oxford: The Cochrane Collaboration; 1994.

21. NHS Centre for Reviews and Dissemination. Undertaking Systematic Reviews of Research on Effectiveness. York: NHS Centre for Reviews and Dissemination, University of York; 2001. Report No.: CRD Report Number 4 (2nd Edition). 
22. Stress incontinence. Clinical Evidence 2004;11:2543-2557.

23. Dickersin K, Scherer R, Lefebvre C. Identifying relevant studies for systematic reviews. BMJ 1994;309(6964):1286-1291.

24. McManus RJ, Wilson S, Delaney BC, Fitzmaurice DA, Hyde CJ, Tobias RS, et al. Review of the usefulness of contacting other experts when conducting a literature search for systematic reviews. BMJ 1998;317(5 December):1562-1563.

25. West S, King V, Carey TS, Lohr KN, McKoy N, Sutton SF, et al. Systems to Rate the Strength of Scientific Evidence. AHRQ Publication No. 02-E016 ed. Evidence Report/Technology Assessment No. 47 (Prepared by the Research Triangle Institute University of North Carolina Evidence-based Practice Center). Rockville, MD: Agency for Healthcare Research and Quality.; 2002.

26. Chiarelli $\mathrm{P}$, Cockburn J. Promoting urinary continence in women after delivery: Randomised controlled trial. BMJ 2002;324(25 May).

27. Dougherty MC, Bishop KR, Abrams RM, Batich CD, Gimotty PA. The effect of exercise on the circumvaginal muscles in postpartum women. Journal of Nurse-Midwifery 1989;34(1):814.

28. Hughes $\mathrm{P}$, Jackson $\mathrm{S}$, Smith $\mathrm{P}$, Abrams $\mathrm{P}$. Can antenatal pelvic floor exercises prevent postnatal incontinence. Neurology and Urodynamics 2001;20(4):447-448.

29. Norton PA, Baker J. Randomized prospective trial of vaginal cones vs Kegel exercises in postpartum primiparous women. Neurology and Urodynamics 1990;9:Abstract 85.

30. Sampselle CM, Miller JM, Mims BL, Delancey JO, Ashton-Miller JA, Antonakos CL. Effect of pelvic muscle exercise on transient incontinence during pregnancy and after birth. Obstetrics and Gynecology 1998;91(3):406-412.

31. Wilson PD, Herbison GP. A randomized controlled trial of pelvic floor muscle exercises to treat postnatal urinary incontinence. International Urogynecology Journal and Pelvic Floor Dysfunction 1998;9(5):257-264.

32. Consort. Intention-To-Treat Analysis. In.

33. Glazener C, Herbison G, Wilson P, MacArthur C, Lang G, Gee H, et al. Conservative management of persistent postnatal urinary and faecal incontinence: randomised controlled trial. British Medical Journal 2001;323(7313):593-596.

34. Morkved S, Bo K, Schei B, Salvesen KA. Pelvic floor muscle training during pregnancy to prevent urinary incontinence: A single-blind randomized controlled trial. Obstetrics \& Gynecology 2003;101(2):313-319.

35. Reilly ETC, Freeman RM, Waterfield MR, Waterfield AE, Steggles P, Pedler F. Prevention of postpartum stress incontinence in primigravidae with increased bladder neck mobility: A randomised controlled trial of antenatal pelvic floor exercises. BJOG: an International Journal of Obstetrics and Gynaecology 2002;109(January):68-76.

36. Henderson JS. Effects of a prenatal teaching program on postpartum regeneration of the pubococcygeal muscle. JOGNN Journal of Obstetric, Gynecologic, \& Neonatal Nursing 1983;12(6):403-408.

37. Morkved S, Bo K. The effect of postpartum pelvic floor muscle exercise in the prevention and treatment of urinary incontinence. International Urogynecology Journal and Pelvic Floor Dysfunction 1997;8(4):217-222.

38. Morkved S, Bo K. Effect of postpartum pelvic floor muscle training in prevention and treatment of urinary incontinence: a one-year follow up. British Journal of Obstetrics \& Gynaecology 2000;107(8):1022-1028.

39. Nielsen CA, Sigsgaard I, Olsen M, Tolstrup M, Danneskiold-Samsoee B, Bock JE. Trainability of the pelvic floor. Acta Obstetricia et Gynecologica Scandinavica 1988;67:437440. 
40. Burgio KL, Zyczynski H, Locher JL, Richter HE, Redden DT, Clark Wright K. Urinary incontinence in the 12-month postpartum period. Obstetrics \& Gynecology 2003;102(6):1291-1298.

41. Chiarelli $P$, Murphy $B$, Cockburn J. Acceptability of a urinary continence promotion programme to women in postpartum. British Journal of Obstetrics \& Gynaecology 2003;110:188-196.

42. Morkved S, Bo K, Fjortoft T. Effect of adding biofeedback to pelvic floor muscle training to treat urodynamic stress incontinence. Obstetrics \& Gynecology 2002;100(4):730-739. 